\title{
'Take-over'-an Unusual Selection Process in Steady-State Cultures of Escherichia coli
}

\author{
By R. J. MUNSON AND B. A. BRIDGES \\ Medical Research Council Radiobiological Research Unit, Harwell, Didcot, Berkshire
}

(Received 6 July 1964)

\begin{abstract}
SUMMARY
When the proportion of prototrophic revertants in a continuous culture of the tryptophan-requiring strain WP2 of Escherichia coli was adjusted to exceed about $10^{-7}$, a large increase ('take-over') often occurred after a variable delay, the proportion becoming as high as $50 \%$ in some cases. The phenomenon appeared to be correlated with a selective advantage of revertants which became attached, with parent auxotrophs, to the wall of the culture tube. After some hours the film of growth on the wall was composed of approximately $10^{7}$ bacteria. It was quite different from the massive 'sticky' growth sometimes encountered in continuous cultures of $E$. coli. The experimental observations of this 'take-over' are quantitatively consistent with a hypothesis of wall adsorption of rather rare and readily attached variant prototrophs.
\end{abstract}

\section{INTRODUCTION}

Turbidimetrically-controlled continuous cultures have proved useful in investigations of both the lethal and mutagenic effects of ionizing radiation (Munson \& McLean, 1961 ; Munson \& Jeffery, 1964). Success when studying mutation induction depends largely on the assumption that there exist no selection pressures operating either for or against the mutants. Such an assumption appears to be valid under most conditions in the present system (Munson \& Jeffery, 1964). Occasionally, however, a rare event occurs which gives a very significant selective advantage to the mutants, resulting in an increase in their number by a factor of $10^{8}$ or more. We have termed this phenomenon 'take-over'. It appears to arise as a result of attachment of bacteria to the walls of the culture tube, the mutant organisms adhering more firmly than non-mutant ones. It is therefore a phenomenon which is of possible importance in any continuous culture maintained for a long period in the same vessel.

\section{METHODS}

Reversions to tryptophan independence in strain WP 2 of Escherichia coli were studied in cultures grown at $37^{\circ}$ in a minimal medium (' $M$ ' of Haas \& Doudney, .1957) supplemented with $6 \mu \mathrm{g}$. tryptophan $/ \mathrm{ml}$. The culture vessels had volumes of approximately $9 \mathrm{ml}$. and the bacterial population was usually maintained at between $10^{8}$ and $2 \times 10^{8}$ bacteria/ml. The proportion of mutants was increased artificially either by exposing the growing culture to gamma radiation, or by adding a known number of prototrophs from a culture derived from revertants which had arisen spontaneously. Viable bacteria were scored after $48 \mathrm{hr}$ incubation at $37^{\circ}$ on the surface of ' $M$ ' medium plates in the case of prototrophs and either plates of 
nutrient agar or ' $\mathrm{M}$ ' medium with $20 \mu \mathrm{g}$. tryptophan $/ \mathrm{ml}$. in the case of total counts of prototrophs and auxotrophs. Where the number of prototrophs was below about $10^{3} / \mathrm{ml}$., a larger volume than the $0.1 \mathrm{ml}$. used for direct plating was passed through a membrane filter and the latter placed on the surface of the agar plate. The growth rate was obtained by measuring the rate at which fresh medium was demanded by the turbidostatic control system. Automatic counting of the drops (of constant but undetermined volume) passing into the culture gave a very sensitive indication of changes in growth rate with time. Measurement of the overflow collected during a known period of time enabled the absolute growth rate to be determined once the volume of the culture vessel was accurately known. The generation time of $\boldsymbol{E}$. coli wP 2 under these conditions was about $43 \mathrm{~min}$. Full details of the continuous culture apparatus, radiation source and microbiological techniques are given elsewhere (Munson \& Jeffery, 1964). Streptomycin-resistant varieties of E. coli wP 2 were obtained by growing the organism in increasing concentrations of the antibiotic. They were assayed on plates containing streptomycin sulphate (Glaxo) $15 \mu \mathrm{g} . / \mathrm{ml}$.

\section{RESULTS}

'Take-over'

In the first experiment (Fig. 1) two cultures were exposed to gamma rays from ${ }^{137} \mathrm{Cs}$ during $4 \mathrm{hr}$ to a total dose of $4500 \mathrm{rads}$, during which time the frequency of prototrophs (try ${ }^{+} /$try $^{+}+$try $\left.^{-}\right)$increased from $10^{-8}$ to $2 \times 10^{-7}$. After a short pause the try ${ }^{+}$frequency in one culture began to increase again and continued at a steady rate until it reached an apparent maximum of about $50 \%$. The other culture showed a similar increase after a longer delay. The growth rates of both cultures during the periods of 'take-over' were constant to within $1 \%$.

Figure 2 shows the changes in try ${ }^{+}$frequency in another experiment which involved two separate irradiations. The first, of 600 rads during $6 \mathrm{hr}$, increased the proportion of prototrophs to about $3 \times 10^{-8}$, at which value it remained constant for $40 \mathrm{hr}$. The second irradiation (600 rads in $6 \mathrm{hr}$ ) increased the value to almost $10^{-7}$ and, very soon after this, 'take-over' occurred, the proportion of prototrophs increasing during $30 \mathrm{hr}$ to just over $10^{-2}$. The constancy of the fraction of prototrophs in the interval between the two radiation exposures confirms the normal absence of selection pressures. Towards the end of the experiment there was a slight increase in growth rate, this being the only occasion in ten cases of 'take-over' when the growth rate altered perceptibly. The final value of try ${ }^{+}$frequency was not always the same from experiment to experiment, but in no case was 'take-over' complete in the sense that all auxotrophs were eliminated.

'Take-over' was also initiated after a variable delay when either previously isolated prototrophic revertants or the parent strain Escherichia coli $\mathrm{B} / \mathrm{r}$ were added to a culture of auxotrophs. The smaller the number of prototrophs added, the greater was the delay observed before 'take-over' started, and when less than 100 prototrophs were added it was not observed at all. Once begun, 'take-over' continued at about the same rate in all experiments.

Cell division was necessary for 'take-over', since a culture which was allowed to become stationary by cutting-off the medium supply showed no increase in the frequency of prototrophs until fresh medium was added (Fig. 3). 


\section{Possible transfer of genetic information}

It seemed possible that the phenomenon might be explained in terms of transfer of genetic information from prototrophs to auxotrophs. Filtrates through membrane filters and chloroform preparations of cultures in the process of active 'takeover' showed no transforming or transducing activity however, and the addition of acridine orange to a culture or to the plating medium did not in any way affect the normal course of 'take-over', indicating that free nucleic acid (e.g. an autonomous episome; Jacob \& Wollman, 1961) was not involved.

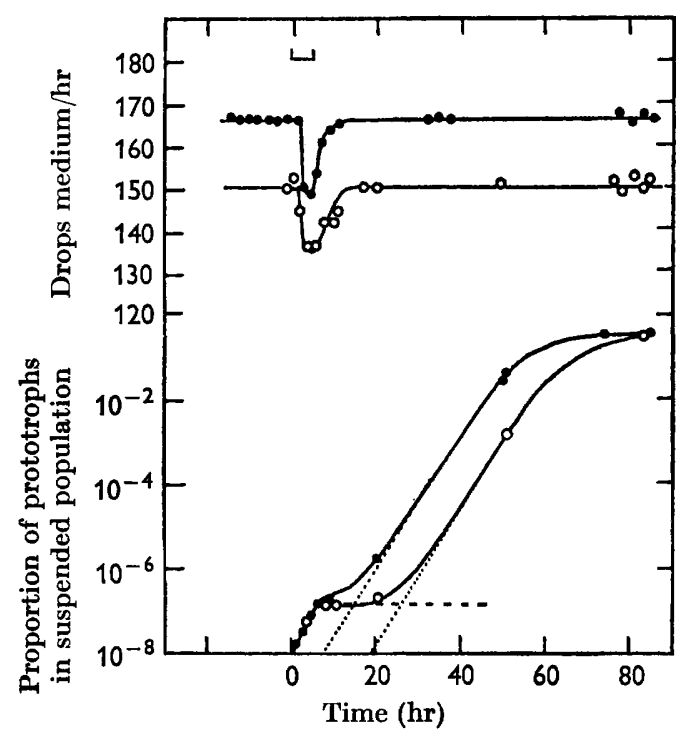

Fig. 1

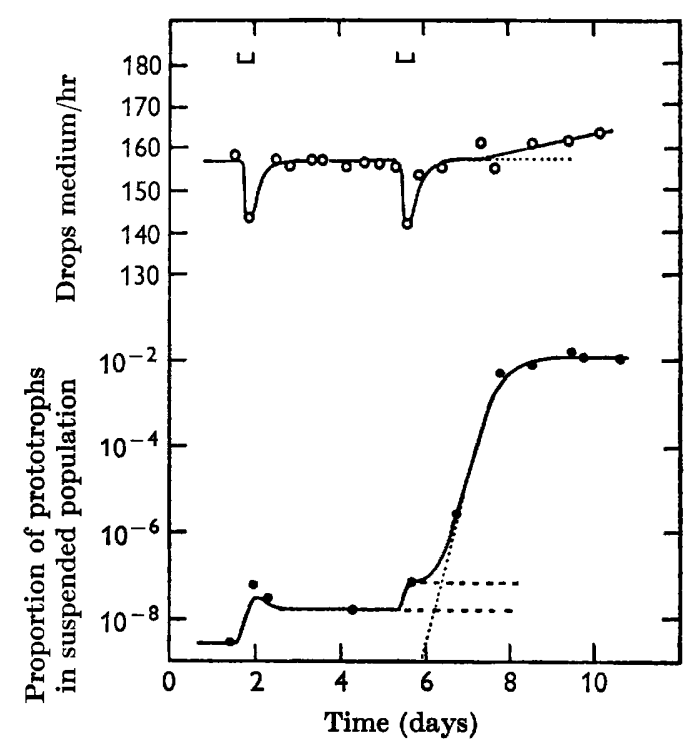

Fig. 2

Fig. 1. Increases in prototroph frequency ('take-over') in two continuous cultures of Escherichia coli wP 2. The cultures were simultaneously exposed to 4500 rads of gamma rays from ${ }^{137} \mathrm{Cs}$ during the period indicated by the heavy horizontal line $(0-4 \mathrm{hr})$. The dashed line indicates the contribution from normal try ${ }^{+}$bacteria and the dotted lines the contributions from the hypothetical variant try ${ }^{+}$bacteria (see text), their slopes being 0.36 hr-1. Observations of medium flow (drops/hr) showed that the growth rates were constant to $\pm 1 \%$ except during and immediately after irradiation.

Fig. 2. 'Take-over' following two irradiations of a culture of Escherichia coli wP2. Two doses each of 600 rads were given during the periods indicated by the heavy horizontal lines. The dotted line, representing the contribution from hypothetical variant try ${ }^{+}$ organisms, has a slope of $\mathbf{0 . 3 5} \mathrm{hr}^{-1}$. After 'take-over' the growth rate (indicated by medium flow, drops $/ \mathrm{hr}$ ) increased slowly by approximately $3 \%$ suggesting that there was an increase in the number of bacteria on the wall equivalent to $3 \%$ of the whole population.

Although conjugation is not known within strains of the Escherichia coli в family (which act as $\mathrm{F}^{-}$in crosses with some $\mathrm{K} 12$ strains; de Haan, 1954) experiments were carried out to test this possibility. Normal streptomycin-sensitive prototrophs were added in the proportion of $10^{-6}$ to streptomycin-resistant auxotrophs. In no instance were streptomycin-resistant prototrophs recovered after 'take-over' had occurred. 


\section{The film of bacteria on the walls of the culture vessels}

When a culture was divided and half of it transferred to a fresh vessel, the course of 'take-over' in the fresh vessel was delayed, indicating that something essential to 'take-over' was left behind in the original vessel (Fig. 4). Tests showed that this was not due to the continuing infection of the original culture by a build-up of prototrophs on the medium input tube or the overflow tube. After a culture in process of 'take-over' was carefully removed from its vessel by Pasteur pipette, a large number of organisms could be washed off the walls and magnetic stirrer by using sterile saline $(\mathrm{NaCl}, 0.85 \%, \mathrm{w} / \mathrm{v})$ and a camel hair brush. The proportion of prototrophs among these wall-adherent organisms was 10 or more times greater than among those in suspension.

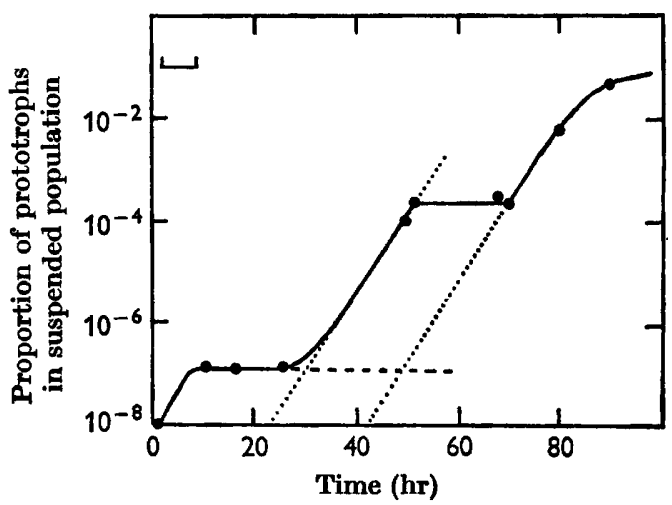

Fig. 3

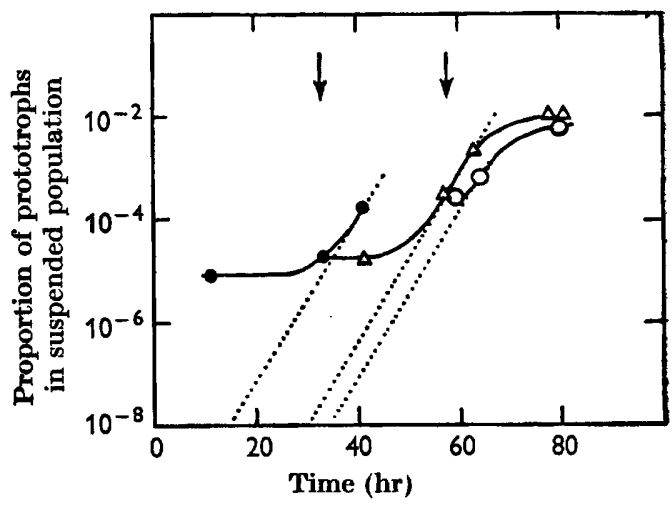

Fig. 4

Fig. 3. 'Take-over' in a culture of Escherichia coli wP2 following exposure to 4500 rads of gamma radiation: dependence upon active growth. The radiation was given during the period indicated by the heavy horizontal line. At $48 \mathrm{hr}$ the supply of medium was stopped until $66 \mathrm{hr}$ and then renewed. During this time of no growth the prototroph frequency did not change but subsequently 'take-over' continued as with similar cultures represented in Fig. 1.

Fig. 4. 'Take-over' in a culture of Escherichia coli wP2 after addition of a small number of prototrophs. Approximately $10^{4}$ prototrophs of a previously isolated spontaneously revertant stock were added at $12 \mathrm{hr}(0)$. At $33 \mathrm{hr}$, half of the culture was transferred to a fresh vessel $(\triangle)$ and, at $56 \mathrm{hr}$, half of the latter culture was put into a third vessel (O). The steepest parts of the curves all have approximately the same slope, viz. $0.35 \mathrm{hr}^{-1}$, and the delay between inoculation and 'take-over' is reduced with each transfer as would be expected on the proposed hypothesis.

Experiments were made on the rate of formation of wall films on clean culture vessels and, although the results were not quantitatively reproducible, the number of organisms in a film increased rapidly in the first few minutes and then more slowly until about $0.7 \%$ of the total population could be washed off the wall after $5 \mathrm{hr}$ of growth in a culture at a population density of $10^{8} / \mathrm{ml}$. Thereafter, the proportion on the wall rose slowly, if at all. These wall films were invisible in the presence of the suspensions but when these were removed the wall films could be seen to cover the whole of the glass surfaces which had been in contact with the cultures. These films were very different from the massive 'sticky' wall growth which occasionally occurred and which, with turbidimetric control of the culture, was 
usually manifested by an increase in apparent growth rate. When encountered, these growths have usually been entirely composed of auxotrophs.

\section{DISCUSSION}

Any postulated mechanism for 'take-over' in Escherichia coli as here described must account for the fact that it happens rarely and apparently randomly, except when large numbers of prototrophs are added or induced, and also that it never proceeds to a prototroph frequency of $100 \%$. The simplest explanation of the data would be that the growth rate of prototrophs is greater than that of auxotrophs; a difference of about $30 \%$ would be necessary to explain the observed rate of 'take-over'. Growth rate of auxotrophs and prototrophs isolated from cultures in process of 'take-over' were, however, identical, whilst the overall growth rate of cultures showed no change even when the proportion of prototrophs approached $50 \%$. Selection due to difference of growth rate is therefore ruled out.

The correlation between the delay before the onset of 'take-over' and the number of prototrophs present might indicate either that a rare spontaneous event occurs among prototrophs which renders one of them able to initiate 'take-over', or that time is required for the build-up of some essential activating substance. The experiments in which cultures were divided, one half being transferred to a fresh vessel, do not rule out the possibility of an activating substance; but they are more simply explained on the hypothesis that time is required for the establishment of try ${ }^{+}$ bacteria of a particular type on the wall. It is clear that, whatever the reason for the selective advantage of prototrophs, the phenomenon is sustained and probably initiated on the wall of the vessel.

Calculation shows that the stirring of the culture at $300 \mathrm{rev} . / \mathrm{min}$. introduces quite appreciable centrifugal forces which tend to thrust bacteria in suspension against the cylindrical wall of the culture vessel. Disregarding local turbulence in the suspension, the centrifugal force on a bacterium $2 \mathrm{~mm}$. from the wall is equal to its weight; but nearer the wall its circumferential speed is decreased by viscous drag until at $1 \mu$ from the wall the suspension is moving at only $10 \mu / \mathrm{sec}$. and the centrifugal force on the bacterium is negligible. Even at this point, however, a bacterium is drifting parallel to the wall at a speed approximately 20 times greater than its mean thermal agitation velocity and a force of the order of $10^{-8}$ dyne would be required to hold it to the wall. Such a force could arise by electrostatic attraction between the bacterium and the wall, the magnitude of this force being critically dependent on the charge distribution on the surface of the bacterium and also on the local microstructure of the glass surface. With reference to the latter it is interesting that the wall (area about $12 \mathrm{~cm} .^{2}$ ) could accommodate $4 \times 10^{9}$ bacteria in a closepacked single layer, whilst the greatest number actually found on the wall was only $1 \%$ of this.

Slow non-turbulent movement of the suspension in contact with the wall film might be expected to allow very poor mixing and so lower the growth rate of organisms there although, with only $0.7 \%$ of the population on the wall, this phenomenon would not be detected by measurements of the growth rate of the whole culture. One experiment in which macroscopic sticky wall growth eventually appeared showed that, at the time of transfer to another vessel, an increase in 
apparent growth rate $(5 \%)$ had taken place which corresponded almost exactly with that to be expected from a number of freely growing bacteria equal to the number brushed from the wall. It therefore seems that the rate of growth of bacteria on the vessel wall was not limited to a significant extent.

Hypothesis of formation of wall film of bacteria. By analogy with the adsorption of gas molecules to a solid surface one can regard the glass wall of the culture tube as providing a finite number $s$ of sites for attachment of a monolayer of bacteria. If at time $t$ a number $n$ of these sites is occupied it may be assumed that the probability of a bacterium in suspension becoming attached between times $t$ and $t+d t$ is $\alpha(s-n) d t$, where the coefficient $\alpha$ is approximately independent of $n$. The probability that in the same time interval a bacterium already attached to the wall will leave it can be written $\beta d t$ where $\beta$ is also approximately independent of $n$.

Let $\bar{\nu}$ and $\bar{\nu}_{a}$ be the number growth rates (Powell, 1956) of bacteria in suspension and in the adsorbed wall film respectively, $D$ the dilution rate and $N$ the total number of bacteria in the culture suspension. The differential equations describing the changes of $N$ and $n$ with time are

$$
\begin{aligned}
& \frac{d N}{d t}=\beta n-N \alpha(s-n)+(\bar{\nu}-D) N, \\
& \frac{d n}{d t}=\left(\bar{\nu}_{a}-\beta\right) n+N \alpha(s-n) .
\end{aligned}
$$

Now the turbidostat automatically keeps $N+n$ constant at a value $C$ say, hence

$$
0=n \bar{\nu}_{a}+N(\bar{\nu}-D) \text {. }
$$

When $n / C$ is small and $n=0$ at $t=0$ equations (1) and (2) have the solution

where

$$
n=C \alpha s[1-\exp (-A t)] / A \text {, }
$$

Observations on the rate of formation of a wall film of try- organisms showed that the initial rate of increase of $n$, viz. $C \alpha s$, was $\sim 2 \times 10^{7} \mathrm{hr}^{-1}$, which with $C$ equal to $2 \times 10^{9}$ makes $\alpha s \sim 10^{-2} \mathrm{hr}^{-1}$. The final value of $n$ as $t \rightarrow \infty$, viz. C $\alpha s / A$, was $7 \times 10^{-3} C$, or $1.4 \times 10^{7}$, so that $A \sim 1.4$. Since one can only assign to $s$ an upper limit of $\sim 4 \times 10^{9}$ and a lower limit of $1 \cdot 4 \times 10^{7}$ it follows from (5) that

$$
1 \cdot 4 \geqslant \beta-\bar{\nu}_{a} \geqslant 0 \text {. }
$$

It has been assumed above that the coefficients $\alpha$ and $\beta$ have the same values for all sites and therefore do not change with $n$ or with $t$ as the sites are filled. In practice some sites will have greater energies for adsorption than others and these will be occupied preferentially so that $\alpha$ will diminish and $\beta$ increase as $n$ increases until eventually $\beta=\bar{\nu}_{a}$. After a very long time an equilibrium will be reached and at this time the right-hand side of equation (2) will become zero and $\beta-\bar{\nu}_{a}$ will reach its maximum positive value.

It is supposed that 'take-over' occurs when a sufficient number of bacteria, which may be one or a very few, of a rather rare try+ variant become attached to the wall of the culture tube. The attachment coefficient $\alpha^{\prime}$ of these variants is assumed to be greater than that of other try- and try+ bacteria, or their detachment coefficient $\beta^{\prime}$ smaller. Their growth rates $\bar{\nu}$ and $\bar{\nu}_{a}$ are assumed to be the same as for try-bacteria. 
Since the film of try- bacteria will generally be fully formed before 'take-over', the variant try + bacteria will compete for the sites of lowest binding energy already occupied by try- bacteria and for others of still lower binding energies for which $\alpha$ is so small and $\beta$ so large that try ${ }^{-}$bacteria are held for very short times only. As a result try ${ }^{-}$bacteria will only remain firmly attached at the sites of greatest adsorption energy and since no further try- attachment can take place at these sites $\alpha=0$ and $\bar{\nu}_{a}=\beta$ at an early stage of 'take-over'. Other sites occupied by try'bacteria will gradually be taken over by the variant try ${ }^{+}$bacteria since $\alpha^{\prime}>\alpha$ and $\beta^{\prime}<\beta$ when $n$ is small. The initial stage of 'take-over' can be described by equations similar to (1) and (2) with the number of variant bacteria on the wall $n^{\prime}$ replacing $n$ and $N^{\prime}$ and $s^{\prime}$ replacing $N$ and $s$, respectively. When $n^{\prime} /\left(s^{\prime}-n-n^{\prime}\right)$ is small one finds

$$
n^{\prime}=C_{1} \exp (\overline{p+q} t)+C_{2} \exp (\overline{p-q} t)+C_{3},
$$

where

$$
\begin{aligned}
p & =\frac{1}{2}\left[\bar{\nu}_{a}-\beta^{\prime}-\alpha^{\prime} \overline{s^{\prime}-n}+\bar{\nu}-D\right], \\
4 q^{2} & =\left[\bar{\nu}_{a}-\beta^{\prime}-\alpha^{\prime} \overline{s^{\prime}-n}+\bar{\nu}-D\right]^{2}+4\left[\left(\bar{\nu}_{a}-\beta^{\prime}\right)\left(\alpha^{\prime} \overline{s^{\prime}-n}+\bar{\nu}-D-\alpha^{\prime} \beta^{\prime} \overline{s^{\prime}-n}\right)\right]
\end{aligned}
$$

and $C_{1}, C_{2}$ and $C_{3}$ are integration constants.

If $\alpha^{\prime} \bar{s}^{\prime}-n \approx \alpha s, p+q \doteq \bar{\nu}_{a}-\beta^{\prime}$ and $(p-q) /(p+q)$ is small and negative. Thus when $t$ is large enough the second and third terms on the right-hand side of (7) can be neglected and

$$
n^{\prime} \doteq C_{1} \exp \left(\overline{\bar{\nu}_{a}-\beta^{\prime}} t\right)
$$

so the graph of $\ln n^{\prime}$ versus $t$ is a straight line of slope $\bar{\nu}_{a}-\beta^{\prime}$. During this stage one can also show that

$$
N^{\prime} \doteq \beta^{\prime} n^{\prime} /\left(\bar{v}_{a}-\beta^{\prime}\right) \text {. }
$$

As $n^{\prime}$ increases $\beta^{\prime}$ will also increase until eventually after a very long time $d n^{\prime} / d t$ becomes zero and then

$$
\left(\bar{\nu}_{a}-\beta^{\prime}\right) n^{\prime}+\left(s-n-n^{\prime}\right) N^{\prime} \alpha^{\prime}=0
$$

so that, if $\alpha^{\prime}>0, \bar{\nu}_{a}-\beta^{\prime} \leqslant 0$.

Since the growth rates of normal and variant bacteria are assumed equal it follows from equation (3) that

$$
n^{\prime} / N^{\prime}=n / N
$$

and so, in the final state when $n$ and $n^{\prime}$ become constant, the proportion of try+ organisms in the suspension, namely $N^{\prime} /\left(N+N^{\prime}\right)$, will never reach $100 \%$ provided some try- bacteria remain attached to the wall.

In the experiments represented in Fig. 1 it is supposed that nearly all of the radiation-induced try ${ }^{+}$bacteria were of the normal type $(\alpha, \beta)$ and so had no selective advantage with respect to the try- bacteria. After the period of irradiation their contribution to the try ${ }^{+}$component remained constant. The few try ${ }^{+}$variants $\left(\alpha^{\prime}, \beta^{\prime}\right)$ present also had no selective advantage until at least one or two became attached to the wall. The time required for attachment is a matter of chance if the number of variants is very small, but since the average proportion of variants amongst the revertants is likely to be constant the time will be shorter when a large increase in number of revertants takes place. The combined frequency of both types of try+ bacteria will thus rise from an initial plateau after an unpredictable latent period until eventually the slope of the graph of $\ln \left(N^{\prime} / N\right)$ versus $t$ becomes equal to $\bar{\nu}_{a}-\beta^{\prime}$. In this way one can account for the random onset of 'take-over'. 
All the experimental graphs of Figs. 1-4 have shapes consistent with the above analysis and maximum gradients of approximately 0.35 in all cases. Since $\bar{\nu}=0.97 \mathrm{hr}^{-1}$ and $\bar{\nu}_{a} \doteq \bar{\nu}$ the values of $\beta^{\prime}$ at this stage are approximately $0.6 \mathrm{hr}^{-1}$. 'Take-over' thus seems to be a consequence of the detachment rate for variant revertants being less than their rate of multiplication, whilst for normal prototrophs on the same surface it is greater.

We thank Professor D. G. Catcheside, Dr D. A. Smith and Dr S. W. Glover for their helpful discussion of the possible involvement of genetic mechanisms. We are indebted to Mr H. Mortimer and Miss Rachel Dennis for technical assistance with some experiments.

\section{REFERENCES}

DE HaAN, P. G. (1954). Genetic recombination of Escherichia coli B. (I). The transfer of the F agent to Escherichia coli в. Genetica, 27, 293.

HaAs, F. L. \& Doudney, C. O. (1957). A relation of nucleic acid synthesis to radiationinduced mutation frequency in bacteria. Proc. nat. Acad. Sci., Wash. 43, 871.

$\mathrm{J}_{\mathrm{ACOB}}$, F. \& Wollman, E. L. (1961). Sexuality and the Genetics of Bacteria. New York: Academic Press Inc.

Munson, R. J. \& JEFFERy, A. (1964). Reversion rate in continuous cultures of an Escherichia coli auxotroph exposed to gamma rays. J. gen. Microbiol. 35, 191.

Munson, R. J. \& Maclean, F. I. (1961). The nature and radiation sensitivity of the long forms of Escherichia coli strain B/r. J. gen. Microbiol. 25, 29.

Powex, E. O. (1956). Growth rate and generation time of bacteria with special reference to continuous culture. J. gen. Microbiol. 15, 492. 